\title{
Letter to the Editor: A response to Kertz (2017): Extension and clarification of a call for more complete reporting and evaluation of experimental methods, physical forms of starters, and results in calf research
}

\author{
A. Bach, ${ }^{*} \dagger^{1}$ M. Terré, $†$ and M. A. Khan $\ddagger$ \\ *ICREA (Institució Catalana de Recerca i Estudis Avançats), 08010 Barcelona, Spain \\ †Department of Ruminant Production, IRTA (Institut de Recerca i Tecnologia Agroalimentàries), 08140 Caldes de Montbui, Spain \\ $\ddagger$ Animal Nutrition and Physiology Team, Grassland Research Centre, AgResearch Limited, Palmerston North, 4442, New Zealand
}

We endorse the first section of the Letter to the Editor in this issue by Kertz (2017) because it makes a relevant call for improving several aspects in the reporting of calf research. We are all in agreement that more basic information is needed. We have attempted, on several occasions, to compile all studies in this area and conduct a meta-analysis but we have failed due to incomplete information, especially concerning the ingredient and nutrient composition of milk replacers. In this regard, the nutrient composition of whole milk should be reported and measured regularly during a study. The type of antibiotic residues potentially present in waste milk and the type and concentration of probiotics (if any) present in milk replacers should be reported because they may affect the microflora of the gastrointestinal tract and ultimately modulate performance. We also support the call for calf studies to collect and report more information on the physical form of solid feeds (e.g., particle size) and on body measurements and health (e.g., diarrhea); however, we think that avoiding citation of articles that do not provide this information is unreasonable and could hinder scientific dialog and progress. In fact, Kertz (2017) refers to several studies about particle size of rations for calves that do not have this information (i.e., Stobo et al., 1966; Coverdale et al., 2004). Furthermore, although we endorse measurements of height and body size and frame, the accuracy of these measures is low and thus we only value them if large sample sizes are considered.

Regarding evaluation of experimental methods and results, particularly those referring to physical form of starters, we should first emphasize that before focusing on the measurements to record and report (e.g., body size, feed particle size distribution), the first premise of every study should be that treatments, per se, are not confounded. For example, 2 articles co-authored by Kertz (Porter et al., 2007; Fokkink et al., 2011) at-

Received November 2, 2016.

Accepted November 13, 2016

${ }^{1}$ Corresponding author: alex.bach@icrea.cat tempted to compare different physical forms of starter feeds but the experimental design was inappropriate because the composition of the starter feeds between treatments was not the same. Porter et al. (2007) compared 2 physical forms and 2 levels of fiber involving 7 to 9 calves per treatment, but the treatments, in addition to differing in particle size, did not have the same nutrient composition. The starter feeds in mash form had 2.88 and 2.73 Mcal of ME/ $\mathrm{kg}$ and 24.5 and $20.7 \%$ $\mathrm{CP}$ for the low and high fiber contents, respectively, whereas the pelleted starter feeds had 2.71 and 2.54 Mcal of ME $/ \mathrm{kg}$ and 22.8 and $24.4 \% \mathrm{CP}$ for the low and high fiber contents. This confounding factor makes it impossible to assess whether the outcomes were due to nutrient supply to calves (e.g., energy was greater in the mash than in the pelleted starter feeds) or due to physical form. The study by Fokkink et al. (2011) compared performance of 4 calves offered a pelleted starter feed with 4 calves fed a texturized starter feed. The texturized feed had $36 \%$ corn and $6 \%$ wheat middlings and did not contain distillers dried grains or soybean hulls, whereas the pelleted starter feed contained $10 \%$ corn, $26 \%$ wheat middlings, $43 \%$ distillers dried grains, and $15 \%$ soybean hulls. As a result, ADF, NDF, fat, and ME differed substantially between treatments. The results from these 2 studies provide no value when assessing the potential effects of particle size because the impact of the differences in feed composition most likely surpassed that due to particle size. On the other hand, the study by Bach et al. (2007) that involved 106 calves is a good example of experimental design and reporting of data, including adequate sample size and particle size distribution of treatments, which shared the exact ingredient and nutrient composition but differed only in physical form (pellet vs. texturized).

Regarding the criticism about Terré et al. (2015), average starter intakes between 8 and $56 \mathrm{~d}$ of life were 510,600 , and $530 \mathrm{~g} / \mathrm{d}$, and ADG were 340, 400 and $360 \mathrm{~g} / \mathrm{d}$ for calves fed pellet starter feed alone, pellet starter feed plus straw, and texturized starter feed alone, respectively. Interestingly, these values were very 
close to those reported by Porter et al. (2007) for the same age period (average intake was $537 \mathrm{~g} / \mathrm{d}$ and ADG was $365 \mathrm{~g} / \mathrm{d})$.

Finally, a clarification: the main message of Kertz (2017), in the second part of the letter, is the danger of not accounting for gut fill when providing forage to calves. We do support that offering large amounts of forage leads to confounded results in growth. However, Castells et al. (2013) reported that total gastrointestinal tract weight was 20.6 and $19.6 \%$ of total BW when no forage was fed and when forage was consumed at $5 \%$ of total solid feed intake, respectively. Furthermore, Khan et al. (2012) reported that heifers fed a starter feed and hay before weaning consumed more feed and had smaller bellies after weaning than calves that had previously received starter feed alone. A growing body of literature exists on this topic, which has recently been thoroughly reviewed by Khan et al. (2016).

In conclusion, to move forward in the area of physical form of the diet offered to young calves, results from the literature should be critically evaluated and integrated without prejudice. Also, when evaluating the outcomes of different treatments in calves, we must not forget that the ultimate goal is weaning the animals and achieve a smooth transition into forage-based rations. A wealth of literature supports the benefit of offering chopped forages early in life in intake, growth, postweaning digestion efficiency, and feeding behavior. However, we agree that caution must be taken when looking at ADG because if forage is consumed in large amounts (i.e., $>5 \%$ of total solid feed consumption), the improvement in growth may simply be an artifact of gut fill, although other outcomes, such as increased intake, cannot be excluded.

\section{REFERENCES}

Bach, A., A. Giménez, J. L. Juaristi, and J. Ahedo. 2007. Effects of physical form of a starter for dairy replacement calves on feed intake and performance. J. Dairy Sci. 90:3028-3033. https://doi. org $/ 10.3168 /$ jds.2006-761.

Castells, L., A. Bach, A. Aris, and M. Terré. 2013. Effects of forage provision to young calves on rumen fermentation and development of the gastrointestinal tract. J. Dairy Sci. 96:5226-5236. https:// doi.org/10.3168/jds.2012-6419.

Coverdale, J. A., H. D. Tyler, J. D. Quigley, and J. A. Brumm. 2004. Effect of various levels of forage and form of diet on rumen development and growth in calves. J. Dairy Sci. 87:2554-2562. https:// doi.org/10.3168/jds.S0022-0302(04)73380-9.

Fokkink, W. B., T. M. Hill, H. G. Bateman II, J. M. Aldrich, R. L. Schlotterbeck, and A. F. Kertz. 2011. Case study: Effect of high and low-cereal-grain starters on straw intake and rumen development of neonatal Holstein calves. Prof. Anim. Sci. 27:357-364.

Kertz, A. F. 2017. Letter to the editor: A call for more complete reporting and evaluation of experimental methods, physical forms of starters, and results in calf research. J. Dairy Sci. 100:851-852. https://doi.org/10.3168/jds.2016-11709.

Khan, M. A., A. Bach, D. M. Weary, and M. A. G. von Keyserlingk. 2016. Invited review: Transitioning from milk to solid feed in dairy heifers. J. Dairy Sci. 99:885-902. https://doi.org/10.3168/ jds.2015-9975.

Khan, M. A., D. M. Weary, D. M. Veira, and M. A. G. von Keyserlingk. 2012. Postweaning performance of heifers fed starter with and without hay during the milk-feeding period. J. Dairy Sci. 95:3970-3976. https://doi.org/10.3168/jds.2011-5027.

Porter, J. C., R. G. Warner, and A. F. Kertz. 2007. Effect of fiber level and physical form of starter on growth and development of dairy calves fed no forage. Prof. Anim. Sci. 23:395-400.

Stobo, I. J. F., J. H. B. Roy, and H. J. Gaston. 1966. Rumen development in the calf. 1 . The effect of diets containing different proportions of concentrates to hay on rumen development. Br. J. Nutr. 20:171-188.

Terré, M., L. Castells, M. A. Khan, and A. Bach. 2015. Interaction between the physical form of the starter feed and straw provision on growth performance of Holstein calves. J. Dairy Sci. 98:1101-1109. https://doi.org/10.3168/jds.2014-8151. 